\title{
Papel del neurotropismo y neuroinvasión de SARS-CoV-2 en la gravedad de los pacientes con COVID-19
}

\author{
Israel Grijalva-Otero*
}

Instituto Mexicano del Seguro Social, Centro Médico Nacional Siglo XXI, Hospital de Especialidades, Ciudad de México, México

\section{Resumen}

La enfermedad (COVID-19) producida por el nuevo coronavirus SARS-CoV-2 se extendió rápidamente desde China a todo el mundo. Aproximadamente una tercera parte de los pacientes infectados de SARS-CoV-2 presenta alteraciones neurológicas, con mayor frecuencia los clasificados como graves que requirieron ventilación mecánica. Por otro lado, casi nueve de cada 10 pacientes admitidos en una unidad de cuidados intensivos no podían respirar espontáneamente, por lo que ameritaron apoyo ventilatorio invasivo y no invasivo. Hasta el momento no se ha determinado si las alteraciones neurológicas tempranas como la hiposmia o anosmia, disgeusia o ageusia, cefalea y vértigo son significativas en la progresión a la forma grave de la enfermedad y se relacionan con la entrada al sistema nervioso central a través de los nervios periféricos. Considerando la gran similitud entre SARS-CoV y SARS-CoV-2 y que la severidad del cuadro que conduce a la muerte no puede ser explicado únicamente por la afección pulmonar, es importante determinar si la invasión potencial del SARS-CoV-2 al sistema nervioso central es parcialmente responsable del componente respiratorio severo que presentan los pacientes con COVID-19.

PALABRAS CLAVE: Insuficiencia respiratoria central. Control central de la respiración. Absorción viral por nervios periféricos. Invasión transináptica.

\section{Role of SARS-CoV-2 neurotropism and neuroinvasion in COVID-19 patients disease severity}

\section{Abstract}

The disease caused by the new SARS-CoV-2 coronavirus (COVID-19) spread rapidly from China to the entire world. Approximately one third of SARS-CoV-2-infected patients have neurological disorders, especially those classified as severe cases and that require mechanical ventilation. On the other hand, almost nine out of 10 patients admitted to an Intensive Care Unit could not breathe spontaneously, thus requiring invasive and non-invasive ventilatory support. So far, whether early neurological disorders such as hyposmia or anosmia, dysgeusia or ageusia, headache and vertigo are significant in the progression to the severe form of the disease or whether they are related to entry to the central nervous system via peripheral nerves has not been determined. Considering the great similarity between SARS-CoV and SARS-CoV-2, and that the severity of the condition that leads to death cannot be explained solely by lung involvement, it is important to determine whether SARS-CoV-2 potential invasion to the central nervous system is partially responsible for the severe respiratory component observed in patients with COVID-19.

KEY WORDS: Central respiratory failure. Central respiratory control. Peripheral nerve viral uptake. Trans-synaptic invasion.

Correspondencia:

*Israel Grijalva-Otero

E-mail: igrijalvao@yahoo.com
Fecha de recepción: 21-05-2020

Fecha de aceptación: 22-06-2020

DOI: 10.24875/GMM.20000307
Gac Med Mex. 2020;156:339-343

Disponible en PubMed

www.gacetamedicademexico.com

0016-3813/৫ 2020 Academia Nacional de Medicina de México, A.C. Publicado por Permanyer. Este es un artículo open access bajo la licencia CC BY-NC-ND (http://creativecommons.org/licenses/by-nc-nd/4.0/). 


\section{Introducción}

A finales de diciembre de 2019, en Wuhan, China, se reportaron grupos de pacientes que presentaban neumonía atípica de origen desconocido. El estudio detallado del lavado bronquial de tres pacientes - que incluyó secuenciación del genoma completo, prueba de reacción en cadena de la polimerasa (PCR, polymerase chain reaction) y cultivo- mostró la presencia de un nuevo virus, al que inicialmente se denominó 2019-nCoV, ${ }^{1}$ perteneciente al género betacoronavirus que incluye a SARS-CoV, bat SARS-like CoV y otros descubiertos en humanos, murciélagos y otros animales salvajes. ${ }^{2}$ La enfermedad producida por este nuevo coronavirus (coronavirus disease [COVID-19]) se extendió rápidamente por el resto del mundo.

Al parecer, la patogénesis de 2019-nCoV (actualmente denominado SARS-CoV-2) es similar a la de SARS-CoV y MERS-CoV, ${ }^{3}$ con el mismo mecanismo de entrada a la célula del hospedero, mediado por el receptor de la enzima convertidora de angiotensina 2 (ACE2, angiotensin-converting enzyme 2). ${ }^{4}$

Los síntomas comunes de COVID-19 al inicio de la enfermedad fueron fiebre, fatiga y tos seca, ${ }^{5,6}$ pero más de la mitad de los pacientes infectados se quejó de disnea y $29 \%$ desarrolló síndrome de insuficiencia respiratoria aguda. ${ }^{6}$

De 26 a $32 \%$ de los casos hospitalizados ameritó ingresar a una unidad de cuidados intensivos ${ }^{5,6}$ y la complicación más frecuente en este grupo fue el síndrome de insuficiencia respiratoria aguda, en $29 \%$ de los casos $(n=12 / 41) .{ }^{6}$ Sin embargo, 32 pacientes (89\%) admitidos en la unidad de cuidados intensivos no podían respirar espontáneamente, por lo que ameritaron apoyo ventilatorio invasivo y no invasivo. ${ }^{5}$

\section{Afectación neurológica por COVID-19}

Numerosos pacientes con COVID-19 han presentado alteraciones neurológicas. Mao et al. identificaron manifestaciones neurológicas inespecíficas (cefalea, deterioro del estado de conciencia, vértigo o convulsiones) y específicas (pérdida del olfato o gusto, alteraciones musculoesqueléticas e infarto cerebral) en $36.4 \%$ de 217 casos; especialmente los trastornos del olfato o del gusto y las alteraciones musculoesqueléticas se presentaron en la fase temprana del curso clínico. ${ }^{7}$ Esos investigadores también registraron mayor frecuencia de síntomas neurológicos en los pacientes que requirieron ventilación mecánica clasificados como graves (45.5\%), que en los no graves (30.2\%); además, los pacientes graves mostraron los datos neurológicos más evidentes, incluidos deterioro del nivel de la conciencia, infarto cerebral y convulsiones. ${ }^{7}$ Otras alteraciones neurológicas como meningoencefalitis ${ }^{8}$ y síndrome de GuillainBarré $^{9}$ han sido relacionadas también con COVID-19.

\section{Probables vías de diseminación al sistema nervioso central}

La presencia de alteraciones neurológicas en aproximadamente una tercera parte de los pacientes infectados de SARS-CoV-2 obliga a tratar de identificar las vías de acceso al sistema nervioso periférico y al sistema nervioso central (SNC). Al ser un padecimiento que afecta a toda la "economía corporal" es esperable que múltiples órganos estén alterados, incluidos los nervios periféricos; sin embargo, el daño al SNC solo es posible si se rompe la barrera hematoencefálica $(\mathrm{BHE})$ o si el virus ingresa por áreas donde no existe la BHE. La vía hematógena, con sus variantes, es utilizada por otros patógenos, ya sea por inflamación de las células endoteliales con ruptura secundaria de la BHE, por los plexos coroides y áreas periventriculares o por inflamación sistémica grave que induce también la ruptura de la BHE. ${ }^{10}$ Otra posibilidad es la vía transetmoidal, mediante la cual los patógenos viajan alrededor de los filetes nerviosos que atraviesan la lámina cribosa, para después diseminarse a través de los espacios de Virchow-Robin alrededor de las arterias y venas que penetran el cerebro."

La sospecha del neurotropismo de SARS-CoV-2 se funda en los síntomas que presentan los pacientes con COVID-19: las alteraciones del olfato y el gusto ${ }^{7,12}$ y los padecimientos específicos como síndrome de Guillain-Barre indican afectación del sistema nervioso periférico;9 el vértigo, la cefalea, el deterioro de la conciencia, las crisis convulsivas o los padecimientos específicos como infartos, hemorragias cerebrales y meningoencefalitis revelan afectación del SNC.7,8

Se ha documentado que la mayoría de los betacoronavirus, incluidos SARS-CoV ${ }^{13}$ y MERS-CoV, ${ }^{14}$ se caracterizan por neurotropismo, el cual les permite afectar al sistema nervioso, a lo que se denomina neuroinvasión. Sin embargo, la infección del SNC no puede ser explicada únicamente por la mediación de la ACE2 (en SARS-CoV) ${ }^{4,15}$ o la dipeptidil peptidasa 4 (en MERS$\mathrm{CoV}),{ }^{16}$ que constituyen los vehículos para ingresar a las células del hospedero, debido a que su expresión en el 
cerebro es muy baja en condiciones normales. ${ }^{15-17}$ De hecho, la ACE2 no se identificó en tejido cerebral sino en el endotelio y músculo liso de los vasos cerebrales. ${ }^{15}$

Dado que SARS-CoV-2 es muy parecido a SARS-CoV, se ha pensado que la afectación al SNC puede seguir las mismas vías. En muestras de pacientes infectados en 2002 y 2003 con SARS-CoV se observaron partículas virales casi exclusivamente en las neuronas. ${ }^{18}$ Posteriormente, en ratones transgénicos infectados intranasalmente se encontró que MERS-CoV y SARS-CoV podían ingresar al cerebro a través de los nervios olfatorios. ${ }^{14,19}$ SARS-CoV se diseminó con rapidez a estructuras cerebrales que se conectan con la vía olfatoria como la corteza piriforme e infralímbica, algunos ganglios basales y el mesencéfalo; así como al tálamo, hipotálamo y amígdalas. También se afectaron otros sitios cerebrales no relacionados con la vía olfatoria como el complejo vagal dorsal, el núcleo ambiguo y el núcleo del hipogloso, lo cual sugiere infección por absorción oral..$^{19}$

En ratones infectados intranasalmente con inóculos de partículas con MERS-CoV, las partículas virales solo fueron detectadas en el cerebro, lo que indicó que la infección del SNC fue más importante en la mortalidad de los ratones infectados..$^{14}$ Asimismo, la infección del centro cardiorrespiratorio del bulbo se presentó aun cuando los ratones recibieron dosis bajas de SARS-CoV, lo que también pudo contribuir a la muerte de los animales. ${ }^{19}$ La relación de la mortalidad con la infección cerebral reportada por Li et al. y Netland et al. en sus modelos experimentales sugiere que la infección del SNC fue más importante que la pulmonar. ${ }^{14,19}$

También se ha demostrado la presencia de los coronavirus en el SNC de seres humanos. En muestras de autopsia de ocho personas que tuvieron síndrome agudo respiratorio severo se encontraron secuencias genómicas de SARS-CoV por inmunohistoquímica, microscopia electrónica y PCR con transcriptasa inversa (RT-PCR), confinadas en neuronas del hipotálamo y la corteza, así como edema y degeneración neuronal en seis de los ocho casos. ${ }^{20}$ En otro caso relacionado con el mismo virus, en el estudio patológico se demostró necrosis neuronal e hiperplasia de células gliales. ${ }^{21}$ Lau et al. describieron a un paciente con crisis convulsivas quien por PCR fue positivo a SARS-CoV en líquido cefalorraquídeo. ${ }^{22}$ Recientemente por RT-PCR se encontró SARS-CoV-2 en el líquido cefalorraquídeo de un paciente con meningitis-encefalitis. ${ }^{8}$

\section{Datos neurológicos y respiratorios en pacientes con coronavirus}

Aunque los pacientes graves con COVID-19 mostraron los datos neurológicos más evidentes (deterioro del nivel de conciencia, infarto cerebral y convulsiones) y los síntomas neurológicos fueron más frecuentes en quienes, además, requirieron ventilación mecánica. ${ }^{7}$ No se ha determinado si las manifestaciones neurológicas específicas (alteraciones del olfato, del gusto y musculoesqueléticas) y las inespecíficas (cefalea y vértigo) desempeñan algún papel en la progresión a la forma grave de la enfermedad.

Por otro lado, más de la mitad de los pacientes reportados por Huang $\mathrm{C}$ et al. se quejó de disnea y casi la tercera parte de ellos desarrolló síndrome de insuficiencia respiratoria aguda. ${ }^{6}$ Además, $89 \%$ de los pacientes admitidos en una unidad de cuidados intensivos reportados por Wang et al. no podía respirar espontáneamente, por lo que ameritó apoyo ventilatorio invasivo y no invasivo. ${ }^{5}$ Finalmente, datos empíricos recopilados en México indican que algunos pacientes sin disnea mostraban desaturación de oxígeno, determinada por oximetría periférica, por lo que fueron hospitalizados y eventualmente intubados (comunicación verbal). Hasta el momento no se ha reportado si la falla respiratoria que ameritó la ventilación asistida en estos pacientes fue tipo 1 o 2; la primera es generada por una neumonía que típicamente desarrolla hipoxia, hipocapnia y aumento de la frecuencia respiratoria, en la que los pacientes pueden respirar espontáneamente, pero con mayor dificultad; en la segunda, la alteración cerebral genera la falla ventilatoria, la cual usualmente se manifiesta con menor frecuencia respiratoria, hipoxia e hipercapnia. ${ }^{23}$ Por lo anterior, no ha podido definirse si el problema respiratorio es central o periférico, o de inicio o predominio en uno u otro.

\section{Neuroinvasión y neurotropismo}

Las crisis convulsivas, infartos y hemorragias cerebrales que presentan los pacientes graves con COVID-19, incluso la meningoencefalitis en el caso señalado, ${ }^{8}$ hacen pensar que la afectación del SNC podría ser generalizada y no focalizada, similar a la generada en otros órganos del cuerpo, lo cual podría atribuirse a invasión por vía hematógena, cuyos mecanismos más probables pudieran ser los siguientes: 
a) Inflamación de las células endoteliales y musculares de los vasos cerebrales con ruptura secundaria de la BHE, dado que a nivel cerebral únicamente las células endoteliales y del músculo liso expresan ACE2. ${ }^{15}$

b) Inflamación sistémica grave o hiperinflamación, caracterizada por el incremento de diferentes citocinas como el factor de necrosis tumoral alfa, interferón gama, diferentes factores estimulantes o quimioatrayentes e interleucinas, entre las que destaca IL-6, ${ }^{24-26}$ que podría inducir también la ruptura de la $\mathrm{BHE}$.

Por otro lado, considerando los síntomas de los pacientes y que los coronavirus como SARS-CoV y MERS-CoV, y muy probablemente SARS-CoV-2, presentan neurotropismo que afecta los nervios periféricos, por estos podrían ingresar al SNC y diseminarse en él en forma transneuronal. ${ }^{14,19}$ Dado que casi nueve de cada 10 pacientes en Europa reportaron disfunción olfatoria y gustatoria, ${ }^{12}$ los nervios olfatorio y glosofaríngeo podrían ser la puerta de entrada al SNC. La absorción olfatoria y oral de los virus en seres humanos y su diseminación transneuronal podrían afectar al complejo vagal dorsal, que incluye el núcleo del tracto solitario y el núcleo ambiguo, estructuras importantes en el ciclo respiratorio. ${ }^{19}$ La absorción viral a través de mecanorreceptores y quimiorreceptores del pulmón y tracto respiratorio también pueden afectar el núcleo del tracto solitario y el núcleo ambiguo. ${ }^{27,28}$ Lo anterior explicaría la falla respiratoria tipo 2 , la cual es posible que influya en la severidad del problema respiratorio y en la mortalidad secundaria. Observaciones empíricas señalan que, al parecer, la neumonía imagenológicamente severa no corresponde a un cuadro clínico respiratorio moderado, ni al tiempo más corto entre la intubación y ventilación mecánicamente asistida y la muerte.

\section{Conclusiones}

La severidad del cuadro que conduce a la muerte no puede ser explicado únicamente por la afectación pulmonar en los pacientes con COVID-19. Debido a la similitud entre SARS-CoV y SARS-CoV-2, resulta importante averiguar si la invasión potencial del SNC por SARS-CoV-2 es parcialmente responsable, para lo cual será necesario demostrar la presencia y distribución topográfica del virus en el tejido cerebral mediante estudios histopatológicos post mortem, así como su detección en líquido cefalorraquídeo por RT-PCR y cultivo en pacientes con complicaciones neurológicas por COVID-19. En pacientes no complicados, pero con síntomas neurológicos, deberán rastrearse biomarcadores de daño neuronal, glial y de la BHE asociados con el diagnóstico de COVID-19, para detectar la invasión temprana al SNC y determinar el papel que desempeña en la gravedad y mortalidad de los pacientes.

\section{Conflicto de intereses}

El autor declara no tener conflicto de intereses.

\section{Financiamiento}

El autor no recibió financiamiento para realizar este artículo.

\section{Responsabilidades éticas}

Protección de personas y animales. El autor declara que para esta investigación no se realizaron experimentos en seres humanos ni en animales.

Confidencialidad de los datos. El autor declara que en este artículo no aparecen datos de pacientes.

Derecho a la privacidad y consentimiento informado. El autor declara que en este artículo no aparecen datos de pacientes.

\section{Bibliografía}

1. Zhu N, Zhang D, Wang W, Li X, Yang B, Song J, et al. A novel coronavirus from patients with pneumonia in China, 2019. N Engl J Med. 2020;382:727-733

2. Tan W, Zhao X, Ma X, Wang W, Niur P, Xu W, et al. A novel coronavirus genome identified in a cluster of pneumonia cases-Wuhan, China 2019-2020. China CDC Weekly. 2020;2:61-62

3. Song Z, Xu Y, Bao L, Zhang L, Yu P, Qu Y, et al. From SARS to MERS, thrusting coronaviruses into the spotlight. Viruses. 2019;11:59.

4. Wan Y, Shang J, Graham R, Baric RS, Li F. Receptor recognition by novel coronavirus from Wuhan: An analysis based on decadellong structural studies of SARS coronavirus. J Virol. 2020;94:e00127-20.

5. Wang D, Hu B, Hu C, Zhu F, Liu X, Zhang J, et al. Clinical characteristics of 138 hospitalized patients with 2019 novel coronavirus-infected pneumonia in Wuhan, China. JAMA. 2020;323:1061-1069.

6. Huang C, Wang Y, Li X, Ren L, Zhao J, Hu Y, et al. Clinical features of patients infected with 2019 novel coronavirus in Wuhan, China. Lancet. 2020:395:497-506.

7. Mao L, Jin H, Wang M, Hu Y, Chen S, He Q, et al. Neurologic manifestations of hospitalized patients with coronavirus disease 2019 in Wuhan, China. JAMA Neurol. 2020;77:1-9.

8. Moriguchi T, Harii N, Goto J, Harada D, Sugawara H, Takamino J, et al. A first case of meningitis/encephalitis associated with SARS-coronavirus-2. Int J Infect Dis. 2020;94:55-58.

9. Toscano G, Palmerini F, Ravaglia S, Ruiz L, Invernizzi P, Cuzzoni MG, et al. Guillain-Barré syndrome associated with SARS-CoV-2. N Engl J Med. 2020:NEJMc2009191.

10. Swanson PA, McGavern DB. Viral diseases of the central nervous system. Curr Opin Virol. 2015;11:44-54.

11. Wehn SM, Heinz ER, Burger PC, Boyko OB. Dilated Virchow-Robin spaces in cryptococcal meningitis associated with AIDS: CT and MR findings. J Comput Assist Tomogr. 1989;13:756-762.

12. Lechien JR, Chiesa-Estomba CM, de Siati DR, Horoi M, le Bon SD, Rodríguez $\mathrm{A}$, et al. Olfactory and gustatory dysfunctions as a clinical presentation of mild-to-moderate forms of the coronavirus disease (COVID-19): A multicenter European study. Eur Arch Otorhinolaryngol. 2020:1-11. 
13. Glass WG, Subbarao K, Murphy B, Murphy PM. Mechanisms of host defense following severe acute respiratory syndromelcoronavirus (SARS-CoV) pulmonary infection of mice. J Immunol. 2004;173: 4030-4039.

14. Li K, Wohlford-Lenane C, Perlman S, Zhao J, Jewell AK, Reznikov LR, et al. Middle East respiratory syndrome coronavirus causes multiple organ damage and lethal disease in mice transgenic for human dipeptidyl peptidase 4. J Infect Dis. 2016;213:712-722.

15. Hamming I, Timens W, Bulthuis ML, Lely AT, Navis G, van Goor H Tissue distribution of ACE2 protein, the functional receptor for SARS coronavirus. A first step in understanding SARS pathogenesis. J Pathol. 2004;203:631-637.

16. Boonacker E, Van Noorden CJ. The multifunctional or moonlighting protein CD26/DPPIV. Eur J Cell Biol. 2003;82:53-73.

17. Harmer D, Gilbert M, Borman R, Clark KL. Quantitative mRNA expression profiling of ACE 2, a novel homologue of angiotensin converting enzyme. FEBS Lett. 2002;532:107-110.

18. Ding Y, He L, Zhang Q, Huang Z, Che X, Hou J, Wang H, et al. Organ distribution of severe acute respiratory syndrome (SARS) associated coronavirus (SARS-CoV) in SARS patients: Implications for pathogenesis and virus transmission pathways. J Pathol. 2004;203:622-630.

19. Netland J, Meyerholz DK, Moore S, Cassell M, Perlman S. Severe acute respiratory syndrome coronavirus infection causes neuronal death in the absence of encephalitis in mice transgenic for human ACE2. J Virol. 2008;82:7264-7275
20. Gu J, Gong E, Zhang B, Zheng J, Gao Z, Zhong Y, et al. Multiple organ infection and the pathogenesis of SARS. J Exp Med. 2005;202:415-424.

21. Xu J, Zhong S, Liu J, Li L, Li Y, Wu X, et al. Detection of severe acute respiratory syndrome coronavirus in the brain: potential role of the chemokine Mig in pathogenesis. Clin Infect Dis. 2005;41:1089-1096.

22. Lau KK, Yu WC, Chu CM, Lau ST, Sheng B, Yuen KY. Possible central nervous system infection by SARS coronavirus. Emerg Infect Dis. 2004:10:342-344.

23. Turtle L. Respiratory failure alone does not suggest central nervous invasion by SARS-CoV-2. J Med Virol. 2020;92:705-706.

24. Fu Y, Cheng Y, Wu Y. Understanding SARS-CoV-2-mediated inflammatory responses: From mechanisms to potential therapeutic tools. Virol Sin. 2020;1-6.

25. Ruan Q, Yang K, Wang W, Jiang L, Song J. Clinical predictors of mortality due to COVID-19 based on an analysis of data of 150 patients from Wuhan, China. Intensive Care Med. 2020;46:846-848.

26. Mehta P, McAuley DF, Brown M, Sanchez E, Tattersall RS, Manson JJ. COVID-19: consider cytokine storm syndromes and immunosuppression. Lancet. 2020;395:1033-1034.

27. Matsuda K, Park CH, Sunden $Y$, Kimura T, Ochiai K, Kida H, et al. The vagus nerve is one route of transneural invasion for intranasally inoculated influenza a virus in mice. Vet Pathol. 2004;41:101-107.

28. Li YC, Bai WZ, Hashikawa T. The neuroinvasive potential of SARSCoV-2 may play a role in the respiratory failure of COVID-19 patients. J Med Virol. 2020;92:552-555. 